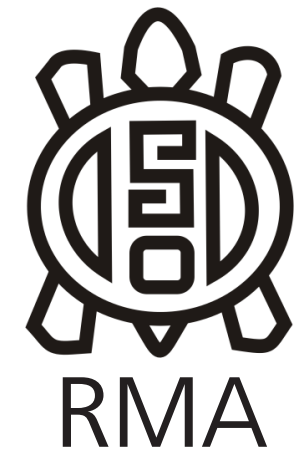

Dossier

\title{
Cazadores recolectores en el bosque de Patagonia y Tierra del Fuego: nuevos datos, patrones y modelos
}

\author{
Hernán Horacio De Angelis* y Juan Bautista Belardi**
}

*Centro Austral de Investigaciones Científicas, CADIC-CONICET. Bernardo Houssay N² 200 (9410). Ushuaia, Tierra del Fuego, Argentina. E-mail: hernandeangelis@yahoo.com.ar

**Universidad Nacional de la Patagonia Austral (ICASUR). CONICET. Campus Universitario, Avda. Gregores y Piloto Lero Rivera s/n. (9400). Río Gallegos, Santa Cruz, Argentina. E-mail: juanbautistabelardi@gmail.com

Los ocho trabajos reunidos en este dossier fueron presentados y discutidos en el simposio "Cazadores recolectores en el bosque de Patagonia y Tierra del Fuego: nuevos datos, patrones y modelos". Se llevó a cabo en el marco del XX Congreso Nacional de Arqueología Argentina, realizado en la ciudad de Córdoba entre los días 15 y 20 de julio de 2019.

La arqueología del bosque es sin duda un tema que ha ido cobrando importancia en la agenda de investigaciones del sur de nuestro país. Las presentaciones y publicaciones a lo largo de los Congresos Nacionales, de las Jornadas de Arqueología de Patagonia y las realizadas en distintas revistas conforman un valioso cuerpo de información sobre el uso del bosque en Patagonia continental y Tierra del Fuego que se consideró importante continuar ampliando y discutiendo.

El objetivo central del simposio fue brindar un ámbito en donde concentrar y actualizar la información generada como resultado de la discusión del papel que habrían cumplido el bosque y el ecotono bosque-estepa del norte y sur de Patagonia y Tierra del Fuego en el marco de las estrategias implementadas por las poblaciones cazadoras recolectoras desde el inicio del ocupaciones hasta momentos históricos. El eje del simposio se focalizó sobre la variabilidad de la señal arqueológica. La disponibilidad de recursos -vegetales y minerales-, el aprovechamiento de la fauna de bosque -huemul y pudú- y del guanaco, la tecnología lítica, el análisis de motivos rupestres, las posibilidades de circulación, las formas, la intensidad y la continuidad del uso del espacio, la interacción entre poblaciones y la manera en que estos ambientes fueron articulados con la estepa, resultaron temas recurrentes a lo largo de todo el simposio. Los trabajos ofrecen los resultados alcanzados por distintos equipos de investigación durante los últimos años y muestran un abanico de enfoques y líneas de análisis que amplían y profundizan el conocimiento sobre las poblaciones cazadoras recolectoras de Patagonia.

El dossier se inicia con el trabajo "Arqueología y Biogeografía humana en el lago Nahuel Huapi: evaluando el rol del ambiente boscoso-lacustre", de Federico L. Scartascini, F. Emmanuel Vargas y Alhue Bay Gavuzzo. Los autores generan un modelo arqueológico con base biogeográfica para el suroeste rionegrino y neuquino durante el Holoceno tardío. La discusión se focaliza en la identificación de variaciones de los registros del arte rupestre de distintos sitios arqueológicos de los ambientes de bosque, ecotono y estepa. Los autores reconocen un paisaje heterogéneo con condiciones de circulación y permanencia muy diferentes.

El segundo trabajo es de Pablo Marcelo Fernández, Cristina T. Bellelli, Mariana Carballido Calatayud, M. Mercedes Podestá, Soledad Caracotche, Sabrina Leonardt, Mercedes G. Fernández y Ana Forlano y se titula "El poblamiento del bosque del centro-norte de la Patagonia argentina: nuevos datos del Parque Nacional Lago Puelo (provincia de Chubut)". Se estudia la heterogeneidad de respuestas en la ocupación humana del ambiente de bosque a través del tiempo y el espacio. Para ello los autores evalúan el alcance del modelo de poblamiento propuesto para el Suroeste de Río Negro - Noroeste de Chubut en el Parque Nacional Lago Puelo-Parque Provincial Río Turbio, haciendo foco en las representaciones rupestres, los modos de circulación humana y en la gestión de los recursos durante el Holoceno tardío. Se destacan similitudes y diferencias con otros espacios de bosque.

El tercer trabajo, presentado por Vivian Scheinsohn, Sabrina Leonardt, Florencia Rizzo y Mercedes G. Fernández, se titula "Cazadores-recolectores y construcción de nicho en el bosque patagónico: el caso del valle del río Pico (Centro-Oeste del Chubut)". Sintetiza las investigaciones arqueológicas desarrolladas en el sector de bosque y ecotono del valle del río Pico. Las autoras analizan los 
factores que podrían haber incidido en el proceso de construcción de nicho de los cazadores-recolectores para el Holoceno tardío y las expectativas arqueológicas que dichos factores generan. Se plantea que el bosque mostraría circuitos de movilidad exploratorios llevados a cabo por poblaciones ubicadas en espacios colonizados con antelación. El trabajo finaliza con el planteo de una agenda de investigaciones.

"Al pie del cerro Fitz Roy: cazadores recolectores en el Noroeste del lago Viedma, provincia de Santa Cruz", de Juan Bautista Belardi, Silvana Laura Espinosa, Flavia Carballo Marina y Rocío Vanesa Blanco, es el cuarto trabajo. Se muestran los resultados de nuevas investigaciones en la localidad de El Chaltén, lindante con el Campo de Hielo Patagónico Sur. Se excavaron sitios en bloques, se realizaron relevamientos de motivos rupestres y se registraron hallazgos aislados. Los autores plantean la integración tardía del bosque a los sistemas cazadores recolectores de la margen Norte del lago Viedma y su uso marginal. Una primera comparación de los resultados con los obtenidos en los ambientes de bosque en las cuencas lindantes de los lagos San Martín y Argentino sugiere la existencia de distintos umbrales de marginalidad.

En el quinto trabajo, "Tecnología lítica y uso del bosque patagónico entre cazadores recolectores: los casos de Puesto Aserradero y Laguna Cóndor (Santa Cruz, Argentina)", María Cecilia Pallo, Natalia Andrea Cirigliano, Judith Emilce Charlin y Luis Alberto Borrero analizan las diferentes formas de uso del ambiente boscoso y ecotono bosque-estepa del suroeste de la provincia de Santa Cruz durante el Holoceno tardío. Se sugiere un escenario más complejo para la ocupación humana que el reconocido previamente. Esto se sostiene a partir del análisis comparativo de dos conjuntos líticos que indican distintas modalidades de uso del bosque por parte de las poblaciones cazadoras recolectoras. Se plantea que los cuerpos de agua, tanto temporarios como permanentes, habrían cumplido un papel importante en dicha diferenciación. Así, distinguen ocupaciones circunstanciales de aquellas recurrentes y planificadas.

El sexto trabajo se titula "Circulación humana durante el Holoceno tardío en el bosque y ecotono bosque-estepa: el curso inferior del río Penitente (suroeste de Santa Cruz)", de Juan Bautista Belardi, Flavia Carballo Marina y Luis Alberto Borrero. Este trabajo ocupa un espacio aledaño al de C. Pallo y colaboradores, por lo que se sugiere leerlos de manera integrada para lograr una mayor perspectiva regional. Se brinda información sobre el sector sur de la zona de los morros, en las nacientes del río Gallegos. El relevamiento de la terraza del río Penitente aportó información sobre tipos artefactuales y materias líticas que son la base para proponer la interacción durante el Holoceno tardío con núcleos poblacionales ubicados en torno a los morros. Se identificó una señal arqueológica relativamente continua a lo largo del río con diversos contextos que muestran variaciones en su intensidad de uso y funcionalidad que sugieren tanto tránsito como mayor permanencia.

La séptima contribución posee un mayor corte metodológico. En "El bosque como fuente de combustible: análisis antracológico de dos sitios ubicados en la faja central de la Isla Grande de Tierra del Fuego", Anna Franch Bach, María Estela Mansur, Vanesa Parmigiani, Hernán De Angelis, María Celina Alvarez Soncini, M. Laura Ciampagna y Aylén Capparelli presentan el análisis antracológico de los materiales arqueológicos de dos sitios de la faja central de la Isla Grande de Tierra del Fuego. A partir del mismo discuten la utilización de recursos leñosos por parte de los grupos cazadores recolectores que habitaron el área durante el Holoceno Tardío y se sugiere el aprovechamiento intenso y variado de los bosques donde se ubican los sitios.

En el último trabajo, "Patrimonio arqueológico e incendios forestales. Las Yeguas, un sitio en la zona sudoccidental del Lago Fagnano", María Celina Alvarez Soncini, Vanesa Parmigiani, Hernán De Angelis, Anna Franch Bach, María Estela Mansur y Carla Pichioni discuten las relaciones de un sitio arqueológico, ubicado en el extremo oeste del lago Fagnano -corazón de la Isla grande de Tierra del Fuego-, con los recursos circundantes y la utilización del bosque como un área de ocupación temporal por parte de las sociedades cazadoras-recolectoras que habitaron el área. El sitio fue identificado luego de un incendio forestal que afecto intensamente la zona y que dejó expuesta una alta frecuencia de materiales arqueológicos. Los resultados amplían el conocimiento sobre el sector occidental del lago Fagnano, a la vez que invitan a pensar acerca de la protección del patrimonio arqueológico.

Agradecemos profundamente a las autoridades del XX Congreso Nacional de Arqueología Argentina por aceptar la propuesta del simposio, a los editores de la Revista del Museo de Antropología, Dres. Roxana Cattáneo y Andrés Izeta, por habernos brindado la oportunidad de publicar los resultados y por toda la ayuda ofrecida durante el proceso de preparación de los trabajos, a Bernarda Conte por las tareas de diseño y a los evaluadores por sus comentarios que ayudaron a precisar la información y las discusiones. Y, por supuesto, a todos los equipos de investigación por su esfuerzo para aportar nuevos datos, reconocer patrones y plantear modelos sobre el uso del bosque de Patagonia y Tierra del Fuego por parte de las poblaciones humanas. 